\title{
Nondestructive control of a mine shaft cast iron concrete lining by active infrared thermography
}

\author{
by M. Zhelnin*, O. Plekhov*, A. Zaicev**, L. Levin**
}

*Institute of Continuous Media Mechanics of the Ural Branch of Russian Academy of Science, 1 Academika Koroleva str., 614013, Perm, Russia, email of corresponding author: poa@icmm.ru

${ }^{* * M i n i n g ~ I n s t i t u t e ~ o f ~ o f ~ t h e ~ U r a l ~ B r a n c h ~ o f ~ R u s s i a n ~ A c a d e m y ~ o f ~ S c i e n c e, ~ P e r m, ~ R u s s i a n ~ F e d e r a t i o n, ~ 78-a ~}$ Sibirskaia str., 614007, Perm, Russia.

\section{Abstract}

The study is devoted to the mathematical modeling of the thermophysical experiment related to diagnostics of the space under cast-iron tubbings of a shaft lining by the optical lock-in thermography. The defect detection is carried out by analyzing of phase characteristics of temperature oscillations on the tubbing surface. To compute the phase characteristics, the digital lock-in correlation method is used. For mathematical processing of the noisy data, the mathematical algorithm has been developed. An effectiveness of the proposed approach has been illustrated by the results of the numerical experiments.

\section{Introduction}

A mine shaft cast iron concrete lining is widely used to support mine shafts under hard geological and hydrogeological conditions [1]. Under using this type of a lining a mine shaft consists of several layers: the cast iron lining, the concrete layer and rock massive. The structural element of the cast iron lining is tubbing. The tubbing is cylindrical segment, which has a smooth outer surface and transverse stiffening ribs on an inner surface.

The reliability of the mine shaft largely depends on the reliability of the lining. For this reason, it is requests to perform diagnostics of the lining to detect defects and cavities located under the tubbing surface. Currently, the lining diagnostics is carried out by a visual inspection. However, the space under the lining cannot be inspected, so instrumental methods have to be used.

It is known that the active infrared thermography is an efficient method for noncontact nondestructive control [26]. The results of the experimental investigations performed in the acting mine shaft of the Uralkali company have shown that this method allows one to implement the defects detection in the concrete layer under the tubbings (figure 1). The hot areas correspond to the cavities located under the tubbing surface.

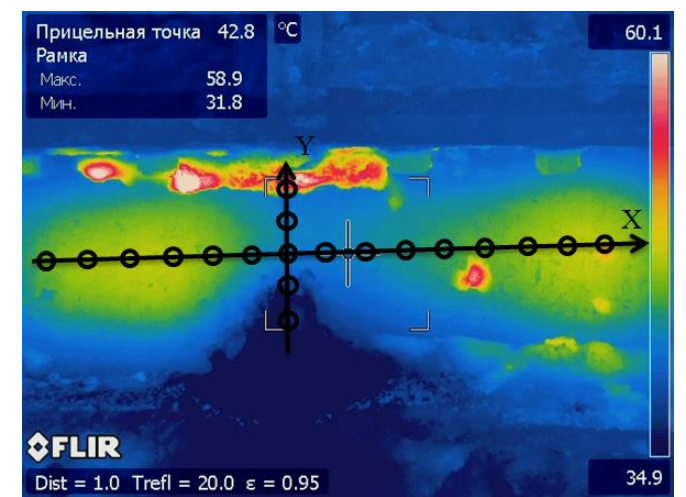

Fig. 1. : The thermogram recorded from the tubbing surface.

The real application conditions requests decreasing of heating time and accompanied by high value temperature noise caused by natural mine shaft conditions. One of the possible solution to increase the efficiency of active infrared thermography if development of numerical algorithms for data treatment. To decease the cost of algorithm testing we developed an additional mathematical model with describe the physics of the problem and allows us to test the data treatment algorithms under natural and extreme temperature noise conditions. The model describes thermophysical experiments of the detection of the water-filled cavity placed under the tubbing by the lock-in thermoghraphy method.

The proposed theoretical model of the experiment includes the heat transfer by conduction, convection and radiation. The analysis of temperature oscillations that aroused on the heating tubbing surface is performed by the digital 
lock-in correlation method. The numerical simulation allows us to determine optimal heating regimes for the defect detection.

To approximate results of the numerical simulation to real experimental conditions, the temperature field distorted by pseudo-random noise. For filtration and smoothing of the noised temperature field, the mathematical algorithm has been developed. The algorithm includes time filtration and smoothing by the Kalman filter and the RTS procedure and spatial smoothing by the smoothing splines method in that the smoothing parameters is estimated by using the $\mathrm{AIC}_{\mathrm{c}}$ criterion.

\section{The mathematical model of the experiment}

The mathematical model of the experiment related to non-destructive testing of a shaft lining by lock-in method is defined as the following heat transfer problem.

$$
\begin{aligned}
& c_{k} \rho_{k} u_{t}=\lambda_{k}\left(u_{x x}+u_{y y}\right), \quad 0<t \leq T,(x, y) \in \Omega_{k}, \quad k=1, \ldots, 4, \\
& \left.u\right|_{t=0}=u_{\mathrm{amb}}, \quad(x, y) \in \bar{\Omega} \\
& -\left.\lambda_{1} \frac{\partial u}{\partial \mathbf{n}}\right|_{\Gamma_{1, \mathrm{~A}}}=q_{0}-q_{1}, \quad-\left.\lambda_{1} \frac{\partial u}{\partial \mathbf{n}}\right|_{\Gamma_{2, \mathrm{~B}}}=0, \quad 0 \leq t \leq T ; \\
& -\left.\lambda_{2} \frac{\partial u}{\partial \mathbf{n}}\right|_{\Gamma_{2, \mathrm{~A}}}=h\left(u-u_{\mathrm{amb}}\right)-q_{2}, \quad 0 \leq t \leq T ; \\
& \left.u\right|_{\Gamma_{2, \mathrm{~B}} \cup \Gamma_{4, \mathrm{~B}}}=u_{\mathrm{amb}}, \quad 0 \leq t \leq T ; \\
& {\left[\left.u\right|_{\bar{\Omega}_{p} \cap \bar{\Omega}_{q}}=0,\left.\lambda_{p} \frac{\partial u}{\partial \mathbf{n}}\right|_{\bar{\Omega}_{p} \cap \bar{\Omega}_{q}}=\left.\lambda_{q} \frac{\partial u}{\partial \mathbf{n}}\right|_{\bar{\Omega}_{p} \cap \bar{\Omega}_{q}}, \quad(p, q) \in\{(2,3),(2,4),(3,4)\}, 0 \leq t \leq T .\right.}
\end{aligned}
$$

$u=u(x, y, t)$ is temperature field within the domain $\Omega$ (figure 2), $t$ is time variable, $\mathbf{n}$ is outer normal, $u_{a m b}$ is the ambient temperature, $c_{k}$ is specific heat capacity, $\rho_{k}$ is density, $\lambda_{k}$ thermal conductivity, $h$ is heat transfer coefficient.

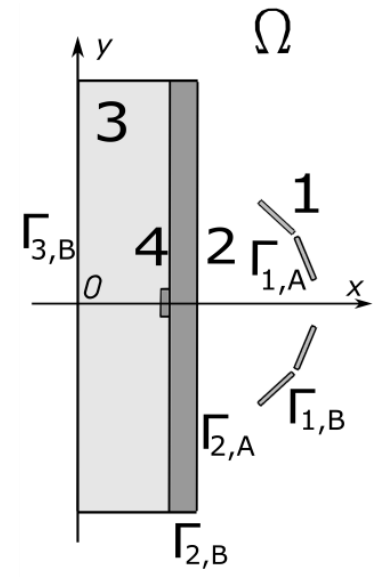

Fig. 2. The geometry of the computational domain $\Omega$. $\Omega_{1}$ - the lamps (1), $\Omega_{2}$ - the cast iron tubbing (2), $\Omega_{3}-$ the concrete layer (3), $\Omega_{4}$ - the water filled cavity (4), $\Gamma_{1, A}$ - front surfaces of lamps, $\Gamma_{1, B}$ - the lateral and rear boundaries of the lamps, $\Gamma_{2, A}$ - the inner boundary of the tubbing, $\Gamma_{2, A}$ - the inner boundary of the tubbing, $\Gamma_{2, B}$ - the lateral and rear boundaries of the tubbing, $\Gamma_{3, B}-$ the lateral and rear boundaries of the concrete layer.

The front surface of lamps is heated by the heat flux:

$$
q_{0}=Q_{0}\left(1-\cos \left[2 \pi f_{e} t\right]\right) / 2(7)
$$

where $Q_{0}$ and $f_{\mathrm{e}}$ are the maximum of heat flux and frequency of heating.

The heat transfers between lamp and tubbing surfaces by surface-to-surface radiation that describes by the heat fluxes $q_{1}$ and $q_{2}$ : 


$$
q_{i}=G_{i}-J_{i}
$$

where $G_{i}$ is irradiation and $J_{i}$ is radiosity.

In order to study an influence of the cavity placed within the concrete layer on the temperature field evolution on the tubbing surface, the analogous problem only without the cavity is solved. For this problem the boundary condition Eq. (6) has the form:

$$
\left.[u]\right|_{\bar{\Omega}_{2} \cap \bar{\Omega}_{3}}=0,\left.\quad \lambda_{2} \frac{\partial u}{\partial \mathbf{n}}\right|_{\bar{\Omega}_{2} \cap \bar{\Omega}_{3}}=\left.\lambda_{3} \frac{\partial u}{\partial \mathbf{n}}\right|_{\bar{\Omega}_{2} \cap \bar{\Omega}_{3}}, \quad 0 \leq t \leq T .
$$

The solution of the problem is denoted by $u_{0}$.

\section{The digital lock-in correlation method}

Suppose that the inner surface tubbing is periodically heated with the frequency $f_{e}$ and the temperature field evolution is registered by an IR camera with the frequency $f_{\text {lock-in }}$ during the time $T$. As a result, the sequence of thermograms are recorded $\left\{\boldsymbol{u}^{(\mathrm{s})}\right\}_{s=1, \ldots, n N}$, where $\boldsymbol{u}^{(\mathrm{s})}=\left(u\left(x_{0}, y_{i}, t_{s}\right), \ldots, u\left(x_{0}, y_{m}, t_{s}\right)\right)$ is the vector of temperature values, $t_{s}=(\mathrm{s}-1) / f_{\text {lock-in }}, n=f_{\text {lock-in/ }} f_{e}$ is the total numbers of thermograms.

To obtain the oscillation component $\left\{\Delta \boldsymbol{u}^{(\mathrm{s})}\right\}_{s=1, \ldots, n N}$ of the temperature, the sequence of thermograms $\left\{\boldsymbol{u}_{0, \text { const }}{ }^{(s)}\right\}_{s=1, \ldots, n N}$ given by the solution $u_{0, \text { const }}$ of the problem without cavity and with the constant heat flux $q_{0}=Q_{0} / 2$ is subtracted from the $\left\{\boldsymbol{u}^{(\mathrm{s})}\right\}_{s=1, \ldots, n N \text {. }}$

The analysis of the thermal signal $\left\{\Delta \boldsymbol{u}^{(\mathrm{s})}\right\}_{s=1, \ldots, n N}$ is carried out by the digital lock-in correlation method that is realized by using a synchronous correlation between the thermal signal and reference harmonic functions [6]. According this method, the in-phase and the quadrature signal is determined for the $p$ oscillation:

$$
\begin{gathered}
S^{0}(i, p)=\frac{1}{n} \sum_{j=1}^{n} \Delta u_{i p j} K_{j}^{0}, \quad S^{\pi / 2}(i, p)=\frac{1}{n} \sum_{j=1}^{n} \Delta u_{i p j} K_{j}^{\pi / 2}, \quad i=1, \ldots, m, p=1, \ldots, N, \\
K_{j}^{0}=2 \sin (2 \pi(j-1) / n), \quad K_{j}^{\pi / 2}=2 \cos (2 \pi(j-1) / n), \quad j=1, \ldots, n,
\end{gathered}
$$

where $\Delta u_{i p j}=\Delta u_{i}\left(y_{i}, t_{s p+j}\right), t_{s p}$ is the time moment from that the $p$ oscillation starts.

To reduce an influence of noise the averaged values are considered:

$$
S_{\mathrm{avr}}^{0}(i)=\frac{1}{N} \sum_{p=1}^{N} S^{0}(i, p), \quad S_{\mathrm{avr}}^{\pi / 2}(i)=\frac{1}{N} \sum_{p=1}^{N} S^{\pi / 2}(i, p), \quad i=1, \ldots, m,
$$

On the basis of the averaged values the amplitude $A(i)$ and the phase of the signal are determined:

$$
A(i)=\sqrt{\left[S_{\mathrm{avr}}^{0}(i)\right]^{2}+\left[S_{\mathrm{avr}}^{\pi / 2}(i)\right]^{2}}, \quad \varphi(i)=\operatorname{arctg}\left(S_{\mathrm{avr}}^{\pi / 2}(i) / S_{\mathrm{avr}}^{0}(i)\right)
$$

It should be noted that each oscillation is studied separately, because it has been established by the numerical simulation that the thermal signal $\left\{\Delta \boldsymbol{u}_{0, \text { const }}{ }_{(\mathrm{s})}\right\}_{s=1, \ldots, n N}$ is not periodical.

\section{The algorithm for filtration and smoothing of the sequence of thermograms distorted by the noise}

In order to estimate an influence of the noise on the analysis of the temperature field evolution the sequence of the sequence of the thermograms $\left\{\boldsymbol{u}_{\text {noise }}{ }^{(\mathrm{s})}\right\}_{S=1, \ldots, n N}$ that is given by adding the pseudorandom white Gaussian noise with zero mean to the sequence $\left\{\boldsymbol{u}^{(\mathrm{s})}\right\}_{s=1, \ldots, n N \text {. }}$

To distinguish the temperature variation aroused due to cavity from the noise, the algorithm for filtration and smoothing of the noised sequence thermograms has been developed. The proposed algorithm includes the following steps:

1) Time filtration of the sequence of thermogram by the Kalman filter

2) Smoothing on the time variable by the Rauch-Tung-Striebel (RTS) procedure.

3) Smoothing on the spatial variables by the smoothing spline methods with using the $\mathrm{AlC}_{\mathrm{c}}$ criterion for estimating the parameter of the smoothing. 
The Kalman filter and the RTS procedure are implemented according to the algorithms presented in [7].

To apply the Kalman filter and the RTS procedure to the sequence of thermogram, it is represented as the linear discrete dynamical system with the process equation:

$$
\boldsymbol{u}^{(s)}=\boldsymbol{F}^{(s, s-1)} \mathbf{u}^{(s-1)}+\boldsymbol{w}^{(s-1)}, \quad s=2, \ldots, n N,
$$

and the measurement equation

$$
\boldsymbol{u}_{\text {noise }}^{(s)}=\boldsymbol{H}^{(s)} \boldsymbol{u}^{(s)}+\boldsymbol{v}^{(s)}, \quad s=2, \ldots, n N
$$

Here $\boldsymbol{F}$ is the transition matrix, $\boldsymbol{H}$ is the measurement matrix, $\boldsymbol{v}^{(\mathrm{s})}, \boldsymbol{w}^{(\mathrm{s})}$ are vectors specifying the process noise.

The initial condition is determined on based of the first recorded thermogram. The matrices $\boldsymbol{F}, \boldsymbol{H}$ are defined

$$
\boldsymbol{F}^{(s, s-1)}=\left(F_{i j}^{(s, s-1)}\right)_{m \times m}, \quad F_{i j}^{(s, s-1)}=\left\{\begin{array}{ll}
\frac{u_{0}\left(x_{0}, y_{i}, t_{s}\right)}{u_{0}\left(x_{0}, y_{i}, t_{s-1}\right)}, & i=j \\
0, & i \neq j
\end{array}, \quad \boldsymbol{H}=\boldsymbol{I} .\right.
$$

where $\left\{\boldsymbol{u}_{0}{ }^{(\mathrm{s})}\right\}_{s=1, \ldots, n N}$ is given by the solution $u_{0}$ of the problem without the cavity and $\boldsymbol{I}$ is the identity matrix.

As mentioned above, the Kalman filter and the RTS procedure allows performing filtration and smoothing on the time variable only. The smoothing on the spatial variables is conducted by the cubic smoothing splines [8]. The degree of the smoothing is controlled by a value of the smoothing parameter $\gamma$.

Consider the set of values $\gamma_{1}<\ldots<\gamma_{M}$ of the smoothing parameter and the corresponding set of the smoothing splines $\left\{g_{1}, \ldots, g_{M}\right\}$. Then according to the $A C_{c}$ criterion [9], the optimal smoothing is given by the value $\gamma_{i}$ for that the parameter aic takes the minimal value:

$$
\operatorname{aic}\left(g_{\lambda}\right)=\ln \frac{\sum_{i=1}^{m}\left\{u_{i}^{(s)}-g_{\lambda}\left(y_{i}\right)\right\}^{2}}{M}+1+\frac{2\left\{\operatorname{tr}\left(\boldsymbol{S}_{\lambda}\right)+1\right\}}{M-\operatorname{tr}\left(\boldsymbol{S}_{\lambda}\right)-2}
$$

where $\boldsymbol{S}_{\mathrm{i}}$ transforms the vector $\boldsymbol{u}^{(\mathrm{s})}$ to the vector $\left(g_{i}\left(y_{1}\right), \ldots, g_{i}\left(y_{m}\right)\right)$.

\section{Results of the numerical simulation}

The results of the numerical simulation of detection of the cavity placed under the tubbing are presented below. The thermophysical properties of the investigated materials are shown in Table 1 . The initial temperature is equal to the ambient temperature $u_{\mathrm{amb}}=283 \mathrm{~K}$, the maximal value of the heat flux on the lamps surfaces is $Q_{0}=20 \mathrm{~kW} \cdot \mathrm{m}^{-2}$, the heat transfer coefficient is $h=10 \mathrm{~W} \cdot \mathrm{m}^{-2} \cdot \mathrm{K}^{-1}$. The geometrical parameters of the domain $\Omega$ are the following. The thicknesses of the concrete layer, the tubbing and the lamps are $0.2 \mathrm{~m}, 0.06 \mathrm{~m}, 0.01 \mathrm{~m}$. The lengths of the tubbing and the lamps are $1.6 \mathrm{~m}, 0.1 \mathrm{~m}$. The distance between the front lamps surfaces and the tubbing surfaces is $0.25 \mathrm{~m}$.

Table 1. The thermophysical properties of the materials

\begin{tabular}{lllll}
\hline \multicolumn{1}{c}{ Parameter } & \multicolumn{1}{c}{ Cast iron } & \multicolumn{1}{c}{ Concrete } & Water & Lamps \\
\hline$c, \mathrm{~kJ} \mathrm{~kg}^{-1} \mathrm{~K}^{-1}$ & 0.5 & 0.89 & 4.2 & 0.4 \\
$\rho \cdot 10^{-3}, \mathrm{~kg} \mathrm{~m}^{-3}$ & 7.2 & 2.4 & 1.0 & 8.7 \\
$\lambda, \mathrm{W} \mathrm{m}^{-1} \mathrm{~K}^{-1}$ & 50 & 1.75 & 0.59 & 400 \\
$\varepsilon$ & 0.9 & & & 0.99 \\
\hline
\end{tabular}

The sequence of the thermograms is extracted from the numerical solution on the inner tubbing surface with the increment $0.005 \mathrm{~m}$ and the frequency $f_{\text {lock-in }}=1$ frame per second. The investigated segment on the tubbing surface has the length $0.4 \mathrm{~m}$, what coincides with the distance between stiffening ribs on the tubbing surface. 


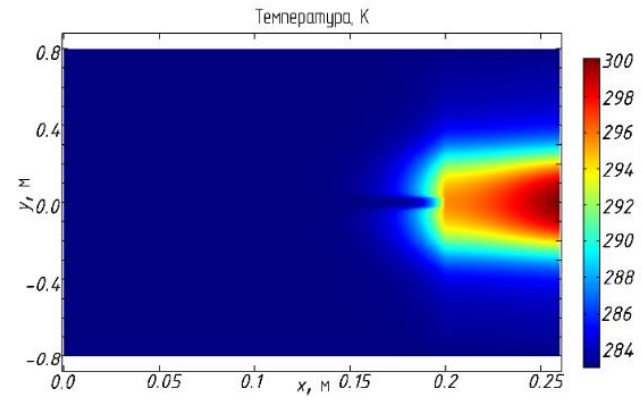

a

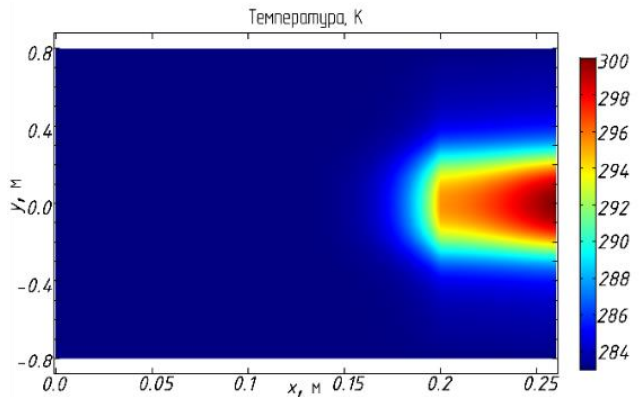

B

Fig. 3. The temperature distributions within the tubbing and the concrete layer with the cavity (a) and without the one (b) at the time moment $t=600 \mathrm{~s}$ and the heating frequency $f_{e}=0.02 \mathrm{~Hz}$.

Figure 3 illustrates the distribution of the temperature field $u$ and $u_{0}$ given by the solutions of (1)-(6) and (1)(5),(9) for the case of heating frequency $f_{e}=0.02 \mathrm{~Hz}$. Form the figure 2 it can be concluded that the cavity impedes the heat propagation from the tubbing to the concrete layer.

In order to determine the optimal value of the heating frequency, the absolute values of phase shifts $\left|\Delta \varphi_{\max }\right|$ between the signals $\left\{\Delta \boldsymbol{u}^{(\mathrm{s})}\right\}_{s=1, \ldots, n N}$ and $\left\{\Delta \boldsymbol{u}_{0}{ }^{(\mathrm{s})}\right\}_{s=1, \ldots, n N}$ are compared:

$$
\Delta \varphi_{\max }=\max _{i=1, \ldots, m} \Delta \varphi(i), \quad \Delta \varphi(i)=\varphi(i)-\varphi_{0}(i),
$$

where phases are calculated from Eqs. (10)-(13).

Figure 4 shows the values of $\left|\Delta \varphi_{\max }\right|$ depending on the heating frequency $f_{e}$. It can be seen, that the maximal absolute value of the phase shift corresponds to $f_{e}=0.02 \mathrm{~Hz}$.

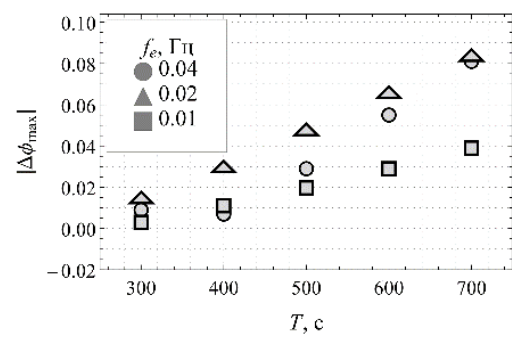

Fig. 4. The maximal absolute value of the phase shift $\left|\Delta \varphi_{\max }\right|$ at the given heating frequency $f_{e}$ in depending on the duration of heating $T$.

In order to investigate an effectiveness of the proposed algorithms of data processing, the two sequences of thermograms $\left\{\boldsymbol{u}_{\text {noise }}{ }^{(\mathrm{s})}\right\}_{s=1, \ldots, n N}$ and $\left\{\boldsymbol{u}_{0, \text { noise }}{ }^{(\mathrm{s})}\right\}_{s=1, \ldots, n N}$ corresponding at $f_{e}=0.02 \mathrm{~Hz}$ are considered under various levels of the noise and durations of the heating $T$.

Figure 5 illustrates results of applying filtration, smoothing and excluding increasing trend to the sequences $\left\{\boldsymbol{u}_{\text {noise }}{ }^{(\mathrm{s})}\right\}_{s=1, \ldots, n N}$ and $\left\{\boldsymbol{u}_{0, \text { noise }}{ }^{(\mathrm{s})}\right\}_{s=1, \ldots, n N}$.

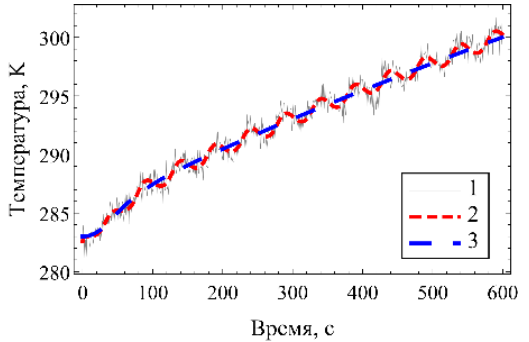

a

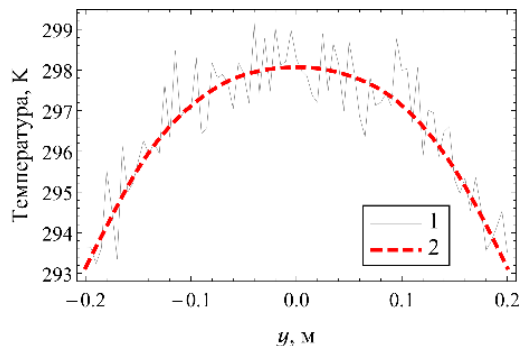

b

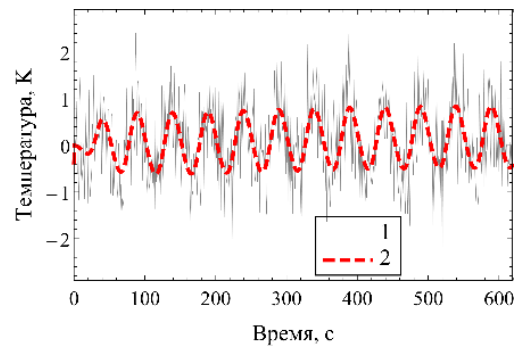

$\mathrm{C}$

Fig. 5. The temperature evolution at a point on the inner tubbing surface (a), the temperature distribution along the inner surface tubbing (b), the temperature oscillations extracted from the curves 1 and 2 (c). The curve 2 is obtained as a result of the applying of the Kalman filter, the RTS procedure and the smoothing spline method to the curve 1, the curve 3 obtained from the solution $u_{0, \text { const }}$ that is used for extracting of the increasing trend.

Figure 6 (a) presents the signal-to-noise ratio (SNR) that is estimated after the sequences $\left\{\boldsymbol{u}_{\text {noise }}{ }^{(\mathrm{s})}\right\}_{s=1, \ldots, n N}$ and $\left\{\boldsymbol{u}_{0, \text { noise }}{ }^{(s)}\right\}_{s=1, \ldots, n N}$ have been processed. The signal-to-noise ratio is defined as 


$$
S N R=\left(\operatorname{avr}\left[\Delta \varphi_{\text {noise }}\right]-\operatorname{avr}\left[\Delta \varphi_{0, \text { noise }}\right]\right) / \sigma_{0},
$$

where $\operatorname{avr}\left[\Delta \varphi_{\text {noise }}\right]$ and $\operatorname{avr}\left[\Delta \varphi_{0, \text { noise }}\right]$ are mean values of phase shifts $\Delta \varphi_{\text {noise }}, \Delta \varphi_{0, \text { noise }}$ calculated by (18) along the investigated segment on the tubbing surface, $\sigma_{0}$ is the standard deviation of $\Delta \varphi_{0 \text {, noise. }}$.

From figure 6 (a) it can be concluded that if the duration of the heating increases, the SNR rises. However, if the noise level increases, the SNR nonlinearly and fast reduces.

Figure $6(\mathrm{~b})$ presents the SNR of the sequences of thermograms obtained under $T=600 \mathrm{~s}$ and noise level 0.8 in depending on the position of the centre of the cavity.

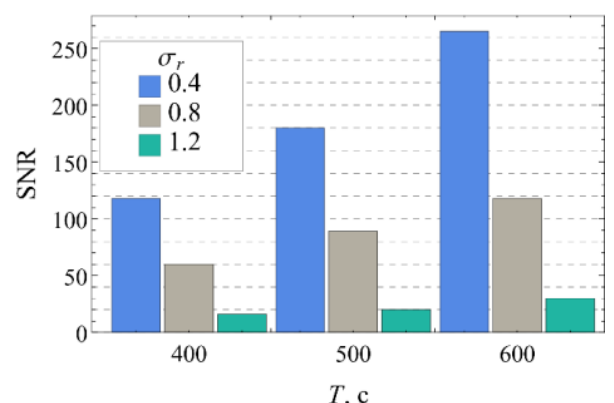

a

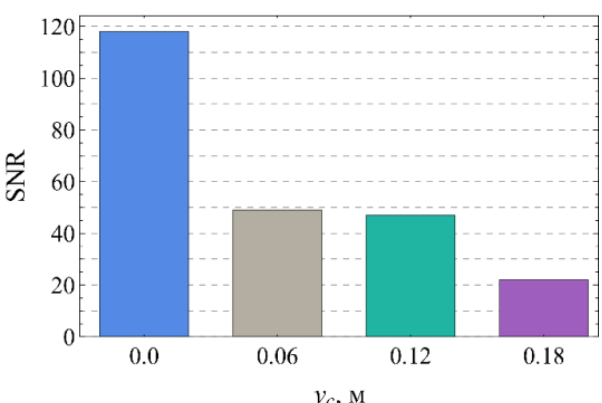

$\mathrm{B}$

Fig 6. The signal-to-noise ratio SNR for the distributions of the phase shifts $\Delta \varphi_{\text {noise, }} \Delta \varphi_{0, \text { noise }}$ at the heating frequency $f_{e}=0.02 \mathrm{~Hz}$ in depending on the heating duration $T$ and the noise level $\sigma_{r}(a)$; in depending on the position of the cavity center $y_{c}$ at $T=600 \mathrm{~s}$ and $\sigma_{r}=0.8(\mathrm{~b})$.

Figure 7 shows the distribution of the phase shifts along the investigated segment under $T=600 \mathrm{~s}$ and noise level 0.8 at the position of the cavity centre $0.0 \mathrm{~m}$ and $0.18 \mathrm{~m}$.

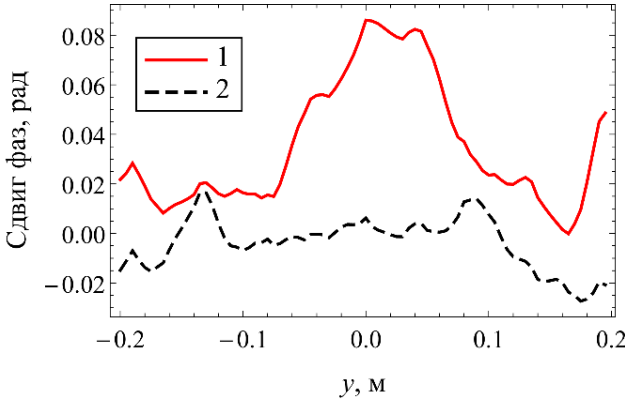

a

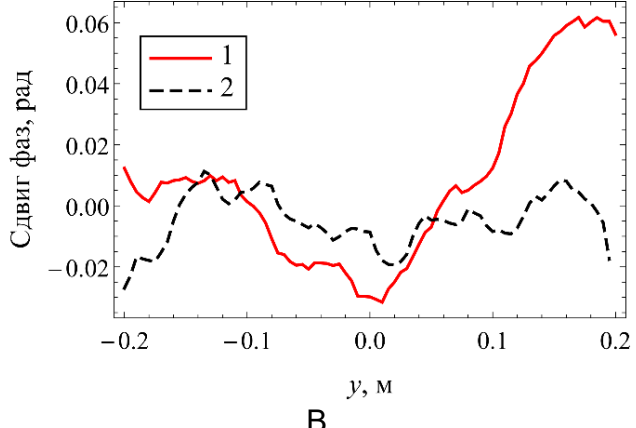

$\mathrm{B}$

Fig. 7. The distribution of the phase shits $\Delta \varphi_{\text {noise }}$ (the curve 1), $\Delta \varphi_{0, \text { noise }}$ (the one 2) at the heating duration $T=$ $600 \mathrm{~s}$ and the noise level $\sigma_{r}=0.8$ for the cases when the center of the cavity coincides the center of the investigated segment $y_{c}=0 \mathrm{~m}$ (a) and when it has been shifted to $y_{c}=0.18 \mathrm{~m}(\mathrm{~b})$.

\section{Conclusions}

On the basis of the numerical simulation it has been established that the lock-in thermography method together with the developed algorithms allows one to detect the water-filled cavity under the tubbing relatively fast and robust.

The detection of the cavity is performed by the analysis of phase shifts of temperature oscillation that is determined by the digital lock-in correlation method. For restoration of the sequence of thermograms distorted by noise, the mathematical algorithm has been proposed. The algorithm includes the Kalman filter, the RTS procedure and the smoothing splines methods in that the value of the smoothing parameters is given by the $\mathrm{AlC}_{\mathrm{c}}$ criterion. The proposed algorithms of data processing allow one to achieve SNR of phase shifts distributions more than 20 under the heating duration $600 \mathrm{~s}$ and the noise level less than 0.8 .

As a result, it has been shown, if the cavity is placed under the tubbing, the splash arises on the phase shift distributions on the tubbing surface. It has been established that for the considered thickness of the tubbing the optimal heating frequency is $0.02 \mathrm{~Hz}$.

\section{REFERENCES}

[1] Olkhovikov YP, The strength of capital excavation of potash and salt mines. Moscow: Nedra; 1984. 
[2] Maldague X. Nondestructive evaluation of materials by infrared thermography. London: Springer; 1993.

[3] Vavilov VP. Thermal/Infrared Nondestructive Testing, NDT Handbook Series. Moscow: Spektrum; 2009.

[4] Busse G., Wu D., Karpen W. Thermal wave imaging with phase sensitive modulated thermography. J. Appl. Phys. 1992; 71(8):3962-3965.

[5] Junyan L., Qingju T., Xun L., Yang W. Research on the quantitative analysis of subsurface defects for nondestructive testing by lock-in thermography. NDT \& E Int. 2012; 45(1):104-110.

[6] Breitenstein O., Warta W., Langenkamp M. Lock-in thermography: Basics and use for evaluating electronic devices and materials. Springer Science \& Business Media, 2010.

[7] Haykin SS. Kalman filtering and neural networks. New York: Wiley; 2001.

[8] P.J. Green, B.W. Silverman. Nonparametric regression and generalized linear models: a roughness penalty approach. CRC Press, 1993.

[9] Lee T. C. M. Smoothing parameter selection for smoothing splines: a simulation study. Comput. Stat. \& Data. Anal. 2003; 42(1): 139-148. 